\title{
Distributed Space-Time Block Codes with Amicable Orthogonal Designs
}

\author{
Trung Q. Duong ${ }^{\dagger}$ and Hoai-An Tran $\ddagger$ \\ ${ }^{\dagger}$ Radio Communications Group, Blekinge Institute of Technology, SE-372 25, Ronneby, Sweden. \\ ${ }^{\ddagger}$ Faculty of Electrical and Electronics Engineering, Ho Chi Minh City University of Transport, Vietnam.
}

\begin{abstract}
In this paper, we generalize the construction of distributed space-time block codes (DSTBCs) using amicable orthogonal designs which are originally applied to co-located multiple-antenna systems. We also derive the closed-form expression of average symbol error probability (SEP). The result is obtained in the form of single finite-range integral whose integrand contains only the trigonometric functions. Using the asymptotic (high signal-to-noise ratio) SEP formulas we show that the orthogonal DSTBCs achieve full diversity order. We also perform Monte-Carlo simulation to validate the analysis.

Index Terms-Distributed space-time block code (DSTBC), non-regenerative relays, orthogonal space-time block code (OSTBC), symbol error probability (SEP).
\end{abstract}

\section{INTRODUCTION}

Without performing any signalling at the relays, repetitionbased cooperative diversity algorithm is the only approach to obtain full spatial diversity with symbol-wise maximum likelihood (ML) decoding over wireless relay networks [1]. In this simple strategy, full spatial diversity order and a single-symbol ML decoding can be obtained with the sacrifice of bandwidth efficiency, because only one relay terminal is allowed to transmit the signals at every sub-channel (time/frequency). Distributed space-time block codes (DSTBCs), i.e., different relays work as co-located transmit antennas and construct a space-time code in a distributed fashion, have been recently proposed to increase data rates for cooperative networks compared with repetition-based scheme [2], [3]. Among cooperation strategies, a non-regenerative or amplify-and-forward (AF) scheme simplifies relaying operation in order to minimize cooperation overhead. In this non-regenerative relay system, relay terminals simply amplify signals and forward to the destination without performing any sort of signal regeneration. Therefore, in this paper, we restrict our attention to the $\mathrm{AF}$ relays.

Recently, single-symbol ML decodable DSTBCs with full diversity order (i.e., the number of relay terminals) have been investigated in [4]. However, these DSTBCs contain a large number of zero entries in the design resulting in a large Peak to Average Power Ratio (PAPR). In [5], the existing orthogonal designs for co-located MIMO system have been applied in AF cooperative networks. It has been showed that these DSTBCs can achieve full diversity order and singlesymbol ML decoding complexity. However, it requires double the number of channel-uses of the first hop, i.e., the source transmitted the signal and its conjugate to the relay at the first and second time-slot of the first hop transmission, respectively. It leads into little decrease of data rate compared to the scheme in [4].

In this paper, we generalize the construction of DSTBCs with amicable orthogonal designs (called orthogonalDSTBCs) originally applied to co-located multiple antennas systems [6], [7] (this approach was first introduced in [5]). Then, it is proved that this scheme achieves the maximum spatial diversity order and single-symbol ML decodability. We also derive closed-form expressions for average symbol error probability (SEP) when the relays are located near by the source. The analytical results are obtained as a single integral with finite limits and an integrand composed solely of trigonometric functions. Using the asymptotic (high signalto-noise ratio) SEP formulas we reconfirm that the orthogonalDSTBC system achieves a diversity order of $K$, i.e., full diversity, where $K$ is the total number of relays. We also perform Monte-Carlo simulations to validate the analysis.

Notation: Throughout the paper, we shall use the following notations. Vector and matrix are written as bold letters. The superscripts $*, T$, and $\dagger$ stand for the complex conjugate, transpose, and transpose conjugate, respectively. $\boldsymbol{I}_{n}$ and $\mathbf{0}_{m n}$ represent the $n \times n$ identity matrix and $m \times n$ zero-entry matrix. $\|\boldsymbol{A}\|_{\mathrm{F}}$ denotes Frobenius norm of the matrix $\boldsymbol{A}$ and $|x|$ indicates the envelope of $x . \mathbb{E}_{x}\{$.$\} is the expectation operator$ over the random variable $x$. A complex Gaussian distribution with mean $\mu$ and variance $\sigma^{2}$ is denoted by $\mathcal{C N}\left(\mu, \sigma^{2}\right) . \Upsilon(\Omega)$ indicates the exponential distribution with hazard rate $\Omega$. log is the natural logarithm. $\Gamma(a, x)$ is the incomplete gamma function defined as $\Gamma(a, x)=\int_{x}^{\infty} t^{a-1} e^{-t} d t$ and $\mathcal{K}_{n}($.$) is$ the $n$ th-order modified Bessel function of the second kind.

\section{Distributed Space-Time Block Codes with Amicable Orthogonal Designs}

We consider a two-hop cooperative diversity protocol with $K$ relays where the channel remains constant for a $T_{\text {coh }}$ coherence time and changes independently to a new value for each $T_{\text {coh. }}$. All terminals are equipped with a single antenna and subject to the half-duplex mode, i.e., a terminal can not transmit and receive simultaneously.

\section{A. First-Hop Transmission: Source-To-Relay}

In the first time-slot, the source transmits $T_{1}$ symbols $\boldsymbol{s}=\left[s_{1}, \cdots, s_{T_{1}}\right]^{T}$ selected from a signal constellation $\mathcal{S}$, with 
average transmit power per symbol $\mathcal{P}_{\mathrm{s}}$. To apply the amicable orthogonal designs as in collocated multiple antennas systems, we double the number of channel uses of the first-hop, during which the source sends the conjugate version of $s$ in the second time-slot [5]. The received signals at the $k$ th relay during the first and second time-slot are given by, respectively

$$
\begin{gathered}
\boldsymbol{r}_{k}^{1}=f_{k} \boldsymbol{s}+\boldsymbol{n}_{k}^{1}, \\
\boldsymbol{r}_{k}^{2}=f_{k} \boldsymbol{s}^{*}+\boldsymbol{n}_{k}^{2},
\end{gathered}
$$

where $\boldsymbol{r}_{k}^{j}, j=1,2$, is the received vector at the $k$ th relay during the $j$ th time-slot, $f_{k} \sim \mathcal{C N}\left(0, \Omega_{f}\right)$ is the Rayleighfading channel coefficient for the source- $k$ th relay link with the channel mean power $\Omega_{f}$, and $\boldsymbol{n}_{k}^{j}$ is complex additive white Gaussian noise (AWGN) of zero mean and variance $N_{0}$.

\section{B. Second-Hop Transmission: Relay-To-Destination}

During the second-hop transmission ( $T_{2}$ symbol-intervals), in order to minimize the signalling cooperation overhead, relays are now working in the AF mode. To simplify relaying operation, a relaying gain is determined only to satisfy the average power constraint with distributional channel state information (CSI) on $h$ (not its realizations) at the relay.

In order to construct a DSTBC, the $k$ th relay multiplies $\boldsymbol{r}_{k}^{1}$ and $\boldsymbol{r}_{k}^{2}$ with $\boldsymbol{A}_{k}$ and $\boldsymbol{B}_{k}$, respectively. Then, these two products is summed up and amplified with a scalar gain $G$ at each relay before forwarding to the destination. Thus, the transmitted signal vector $\boldsymbol{x}_{k}$ from the $k$ th relay is as follows

$$
\begin{aligned}
\boldsymbol{x}_{k} & =G\left(\boldsymbol{A}_{k} \boldsymbol{r}_{k}^{1}+\boldsymbol{B}_{k} \boldsymbol{r}_{k}^{2}\right) \\
& =G f_{k}\left(\boldsymbol{A}_{k} \boldsymbol{s}+\boldsymbol{B}_{k} \boldsymbol{s}^{*}\right)+G\left(\boldsymbol{A}_{k} \boldsymbol{n}_{k}^{1}+\boldsymbol{B}_{k} \boldsymbol{n}_{k}^{2}\right),
\end{aligned}
$$

where $\boldsymbol{A}_{k}$ and $\boldsymbol{B}_{k}$ are $T_{2} \times T_{1}$ matrices and (3) follows immediately from (1) and (2). Assume the coherence time of $g_{k}$ is greater than $T_{2}$, the received signal vector $\boldsymbol{y}_{D}$ at the destination is give by

$$
\begin{aligned}
& \boldsymbol{y}_{D}=\sum_{k=1}^{K} g_{k} \boldsymbol{x}_{k}+\boldsymbol{n}_{D}=\sum_{k=1}^{K} G g_{k} f_{k}\left(\boldsymbol{A}_{k} \boldsymbol{s}+\boldsymbol{B}_{k} \boldsymbol{s}^{*}\right) \\
& +\sum_{k=1}^{K} G g_{k}\left(\boldsymbol{A}_{k} \boldsymbol{n}_{k}^{1}+\boldsymbol{B}_{k} \boldsymbol{n}_{k}^{2}\right)+\boldsymbol{n}_{D},
\end{aligned}
$$

where (4) follows immediately from (3), $g_{k} \sim \mathcal{C N}\left(0, \Omega_{g}\right)$ is the Rayleigh-fading channel coefficient for the $k$ th relaydestination link with the channel mean power $\Omega_{g}$, and $\boldsymbol{n}_{D}$ is AWGN at the destination of zero mean and variance $N_{0}$. Denote $\mathcal{C}_{k}, \boldsymbol{H}$, and $\boldsymbol{N}$ as follows

$$
\begin{aligned}
& \mathcal{C}_{k}=\boldsymbol{A}_{k} \boldsymbol{s}+\boldsymbol{B}_{k} \boldsymbol{s}^{*}, \\
& \boldsymbol{H}=\left[G f_{1} g_{1}, \cdots, G f_{K} g_{K}\right]^{T}, \\
& \boldsymbol{n}=\sum_{k=1}^{K} G g_{k}\left(\boldsymbol{A}_{k} \boldsymbol{n}_{k}^{1}+\boldsymbol{B}_{k} \boldsymbol{n}_{k}^{2}\right)+\boldsymbol{n}_{D},
\end{aligned}
$$

then we can rewrite (4) in the matrix form

$$
\boldsymbol{y}_{D}=\mathcal{C H}+\boldsymbol{n},
$$

where $\mathcal{C}=\left[\mathcal{C}_{1}, \cdots, \mathcal{C}_{K}\right]$, i.e., $\mathcal{C}_{k}$ is the $k$ th column of matrix C.

Now, we consider the construction of $\boldsymbol{A}_{k}$ and $\boldsymbol{B}_{k}$ using the amicable orthogonal designs [6], [7]. It is obvious that the $k$ th relay transmits the corresponding $k$ th column of matrix $\mathcal{C}$. From (5), it is easy to see that $\boldsymbol{A}_{k}$ conveys the entries $s_{n}\left(n=1, \cdots, T_{1}\right)$ and $\boldsymbol{B}_{k}$ conveys the entries $s_{n}^{*}$ on the $k$ th column of $\mathcal{C}$. More specifically, if $\mathcal{C}_{t, k}= \pm s_{n}$ then the $(t, n)$ th entry of $\boldsymbol{A}_{k}= \pm 1$, if $\boldsymbol{C}_{t, k}= \pm s_{n}^{*}$ then the $(t, n)$ th entry of $\boldsymbol{B}_{k}= \pm 1$, otherwise entries of $\boldsymbol{A}_{k}$ and $\boldsymbol{B}_{k}$ are equal to 0 . We now summarize these operations in the following

$$
\text { the }(t, n) \text { th entry of } \boldsymbol{A}_{k}=\left\{\begin{array}{cr} 
\pm 1 & \text { if } \mathcal{C}_{t, k}= \pm s_{n} \\
0 & \text { elsewhere }
\end{array}\right.
$$

and

$$
\text { the }(t, n) \text { th entry of } \boldsymbol{B}_{k}=\left\{\begin{array}{cr} 
\pm 1 & \text { if } \mathcal{C}_{t, k}= \pm s_{n}^{*} \\
0 & \text { elsewhere }
\end{array}\right.
$$

where $t=1, \cdots, T_{2}, n=1, \cdots, T_{1}$, and $k=1, \cdots, K$.

Here are two examples of the codes $\mathcal{G}_{2}$ with rate 1 and $\mathcal{G}_{4}$ with rate $3 / 4$ [6]:

- For the code $\mathcal{G}_{2}$, we need $T_{1}=2, T_{2}=2, K=2$, and

$$
\begin{aligned}
& \boldsymbol{A}_{1}=\left[\begin{array}{ll}
1 & 0 \\
0 & 0
\end{array}\right], \quad \boldsymbol{B}_{1}=\left[\begin{array}{cc}
0 & 0 \\
0 & -1
\end{array}\right], \boldsymbol{A}_{2}=\left[\begin{array}{ll}
0 & 1 \\
0 & 0
\end{array}\right], \\
& \boldsymbol{B}_{2}=\left[\begin{array}{ll}
0 & 0 \\
1 & 0
\end{array}\right] .
\end{aligned}
$$

- For the code $\mathcal{G}_{4}$, we need $T_{1}=3, T_{2}=4, K=4$ (for the case of 4 relays), and $\boldsymbol{A}_{k}, \boldsymbol{B}_{k}$ are given in [5]. Noting that, amicable orthogonal design has "scale-free" property in the sense that it still achieves a large diversity gain when some of the columns are deleted. In other words, when some of the relays are not working we still get a large diversity order by removing some of the $\boldsymbol{A}_{k}$ and $\boldsymbol{B}_{k}$ matrices. For examples, for the case of 2 and 3 relays we have $\boldsymbol{A}_{3}=\boldsymbol{B}_{3}=\boldsymbol{A}_{4}=\boldsymbol{B}_{4}=\mathbf{0}_{43}$ and $\boldsymbol{A}_{4}=\boldsymbol{B}_{4}=\mathbf{0}_{43}$, respectively.

\section{Symbol ERror Probability AND Diversity ORDER}

From (7), we can see that the noise $\boldsymbol{n}$ is a Gaussian random vector with zero mean and the covariance matrix $\boldsymbol{R}=\mathbb{E}\left\{\boldsymbol{n n}^{\dagger}\right\}=\alpha \boldsymbol{I}_{T_{2}}$, where $\alpha=N_{0}\left(\sum_{k=1}^{K} G^{2}\left|g_{k}\right|^{2}+1\right)$. Assuming the destination knows channel information for all links, it is obvious that $\boldsymbol{y}_{D} \mid \boldsymbol{H}$ is a Gaussian random vector with mean vector $\mathcal{C H}$ and covariance matrix $\boldsymbol{R}$. Hence, the ML decoding can be readily written as [8]

$$
\hat{\boldsymbol{c}}=\underset{\mathcal{C}}{\arg \min }\left\|\boldsymbol{R}^{-1 / 2}\left(\boldsymbol{y}_{D}-\mathcal{C} \boldsymbol{H}\right)\right\|_{\mathrm{F}}^{2}
$$

where the minimization is performed over all possible codeword matrices $\mathcal{C}$. Due to the orthogonality of $\mathcal{C}$ 's columns, the ML decoding can be decomposed into a sum of $T_{1}$ terms, where each term depend only one complex symbol $s_{n}, n=1, \cdots, T_{1}$ [9], [10]. Therefore, the minimization of (11) is equivalent to minimizing each decision metric for $s_{n}$ 
separately and the ML receiver selects $\hat{s}_{n}$ for $s_{n}$ if and only if [10]

$$
\hat{s}_{n}=\underset{\tilde{s}_{n} \in \mathcal{S}}{\arg \min }\left|\left(\|\boldsymbol{H}\|_{\mathrm{F}}^{2} s_{n}+\eta\right)-\|\boldsymbol{H}\|_{\mathrm{F}}^{2} \tilde{s}_{n}\right|^{2},
$$

where $\tilde{s}_{n}$ and $\eta \sim \mathcal{C N}\left(0, \alpha\|\boldsymbol{H}\|_{\mathrm{F}}^{2}\right)$ are the combiner output and Gaussian noise for post space-time block decoding, respectively. From (12), we see that the distributed orthogonal space-time block encoding and decoding transform a MIMO non-regenerative relay fading channel into an equivalent single-input single-output (SISO) Gaussian channel with a channel gain of $\frac{1}{\alpha}\|\boldsymbol{H}\|_{\mathrm{F}}^{2}$. The instantaneous signal-to-noise ratio (SNR) per symbol $\gamma$ of the equivalent SISO model is given by

$$
\gamma=\frac{\mathcal{P}_{\mathrm{s}}}{\alpha}\|\boldsymbol{H}\|_{\mathrm{F}}^{2}=\operatorname{SNR} \frac{\sum_{k=1}^{K} G^{2}\left|f_{k}\right|^{2}\left|g_{k}\right|^{2}}{\sum_{k=1}^{K} G^{2}\left|g_{k}\right|^{2}+1},
$$

where SNR $=\frac{\mathcal{P}_{\mathrm{s}}}{N_{0}}$ is the common SNR of each link without fading and (13) follows immediately from the expansion of squared-Frobenius norm of $\boldsymbol{H}$.

Now let's discuss the amplifying gain $G$ and the average transmit power at every relay. Since $f_{k}, n_{k}^{1}$, and $n_{k}^{2}$ are independent complex Gaussian random variables with variance $\Omega_{f}, N_{0}$, and $N_{0}$, respectively, the average transmitted power at relay $k$ is

$$
\mathbb{E}\left\{\left\|\boldsymbol{x}_{k}\right\|_{\mathrm{F}}^{2}\right\}=G^{2}\left(\Omega_{f} T_{1} \mathcal{P}_{\mathrm{s}}+N_{0}\right) .
$$

In general, the optimal power allocation is feasible when the source has the channel knowledge of the entire network. However, such knowledge requires a considerable overhead, and hence, not suitable for our scheme. We apply the equalpower allocation to the source and the relays, i.e., the relays consume a half of total transmit power and each relay consumes an equal amount of power. Hence, we have the following formula: $\mathbb{E}\left\{\left\|\boldsymbol{x}_{k}\right\|_{\mathrm{F}}^{2}\right\}=\frac{1}{K} \mathbb{E}\left\{\|\boldsymbol{s}\|_{\mathrm{F}}^{2}\right\}$. Due to the fact that $\mathbb{E}\left\{\|\boldsymbol{s}\|_{\mathrm{F}}^{2}\right\}=T_{1} \mathcal{P}_{\mathrm{s}}$, the amplifying gain $G$ can be derived as $G^{2}=\frac{1}{K}\left(\Omega_{f}+\frac{1}{T_{1} \mathrm{SNR}}\right)^{-1}$.

We can see that the SEP performance of orthogonal-DSTBC over AF relay channels is completely characterized by the statistical behavior of the instantaneous SNR for each singleinput single-output (SISO) sub-channel. However, it is very difficult to examine the statistical characteristic of $\gamma$ given in (13). For the sake of simplicity, we restrict our attention into the case when the relays are located near by the source. If the relays are much closer to the source than destination, the following approximation may be hold: $\sum_{k=1}^{K} G^{2}\left|g_{k}\right|^{2}+1 \stackrel{\text { high SNR }}{\approx} 1$. In this special case, (13) can be readily written as

$$
\gamma=\operatorname{SNR} \sum_{k=1}^{K} G^{2}\left|f_{k}\right|^{2}\left|g_{k}\right|^{2} .
$$

Since $\mathrm{A}_{k} \sim \mathcal{C N}\left(0, \Omega_{\mathrm{A}}\right), \mathrm{A} \in\{f, g\}$ and $k=1, \cdots, K$, it is obvious that $\left|A_{k}\right|^{2}$ obeys an exponential distribution with hazard rate $1 / \Omega_{\mathrm{A}}$, denoted as $\left|\mathrm{A}_{k}\right|^{2} \sim \Upsilon\left(1 / \Omega_{\mathrm{A}}\right)$. Since $f_{k}$ and $g_{k}$ are statistically independent, the moment generating function (MGF) of $\gamma$, defined as $\phi_{\gamma}(\nu) \triangleq \mathbb{E}_{\gamma}\{\exp (-\nu \gamma)\}$, is given by

$$
\begin{aligned}
& \phi_{\gamma}(\nu)=\mathbb{E}_{f_{k}, g_{k}}\left\{\prod_{i=1}^{K} \exp \left(-G^{2} \operatorname{SNR} \nu\left|f_{k}\right|^{2}\left|g_{k}\right|^{2}\right)\right\} \\
& =\left[\int_{0}^{\infty} \exp \left(-G^{2} \operatorname{SNR} \nu t\right) p_{T}(t) d t\right]^{K} \\
& =\left[\int_{0}^{\infty} \frac{2}{\Omega_{f} \Omega_{g}} \exp \left(-G^{2} \mathrm{SNR} \nu t\right) \mathcal{K}_{0}\left(2 \sqrt{\frac{t}{\Omega_{f} \Omega_{g}}}\right) d t\right]^{K} \\
& =[\lambda \exp (\lambda) \Gamma(0, \lambda)]^{K}
\end{aligned}
$$

where $t=\left|f_{k}\right|^{2}\left|g_{k}\right|^{2}, \lambda=\left(G^{2} \operatorname{SNR} \nu \Omega_{f} \Omega_{g}\right)^{-1}$, (17) follows immediately from [11, Theorem 3], and (18) can be obtained by changing the variable $v=G^{2} \mathrm{SNR} \nu t$ along with [12, eq. (8.353.4)]. Using the well-known MGF approach [13], we obtain the average SEP of the orthogonal-DSTBC scheme with $M$-PSK in relay channels as ${ }^{1}$

$$
\begin{aligned}
P_{\mathrm{e}} & =\frac{1}{\pi} \int_{0}^{\pi-\frac{\pi}{M}} \phi_{\gamma}\left(\frac{g}{\sin ^{2} \theta}\right) d \theta \\
& =\frac{1}{\pi} \int_{0}^{\pi-\frac{\pi}{M}}\left[\zeta \sin ^{2} \theta \times \exp \left(\zeta \sin ^{2} \theta\right) \Gamma\left(0, \zeta \sin ^{2} \theta\right)\right]^{K} d \theta
\end{aligned}
$$

where $g=\sin ^{2}(\pi / M)$ and $\zeta=\left(G^{2} \operatorname{SNR} g \Omega_{f} \Omega_{g}\right)^{-1}$. It can be clearly seen that the SEP is given in exact closed-form expressions in the form of single finite-range integral whose integrand contains only the trigonometric functions. This result can be readily calculated by common mathematical software packages such as MATHEMATICA or MAPLE.

We now assess the effect of cooperative diversity on the SEP behavior in a high-SNR regime. The diversity impact of non-regenerative cooperation on a high-SNR slope of the SEP curve can be quantified by the following theorem.

Theorem 1 (Achievable Diversity Order): The nonregenerative cooperation of orthogonal-DSTBC scheme provides maximum diversity order, i.e.,

$D \triangleq \lim _{\mathrm{SNR} \rightarrow \infty} \frac{-\log P_{\mathrm{e}}}{\log (\mathrm{SNR})}=K$.

Proof: The diversity has been defined as the absolute values of the slopes of the SEP curve plotted on a log-log scale in high SNR regime. As seen from (19), the SEP is expressed in a form of finite integral whose integrand is the MGF of random variable $\gamma$. Therefore, the asymptotic behavior of the MGF $\phi_{\gamma}(\nu)$ at large SNR reveals a high-SNR slope of the

\footnotetext{
${ }^{1}$ The result can be applied to other binary and M-ary signals in a straightforward way (see, e.g., [13]).
} 
SEP curve, we have [14]

$$
D=\lim _{\mathrm{SNR} \rightarrow \infty} \frac{-\log \phi_{\gamma}(g)}{\log (\mathrm{SNR})} .
$$

From (18) and due to the fact that $G^{2} \stackrel{\text { high } \stackrel{\sim}{\approx}}{\approx} \frac{1}{K \Omega_{f}}$, the diversity order can be written as

$$
\begin{aligned}
D & =-K[\underbrace{\lim \frac{\log \left(\frac{\xi}{x}\right)}{\log x}}_{\stackrel{(a)}{=}-1}+\underbrace{\left.\lim _{x \rightarrow \infty} \frac{\log \left(\exp \left(\frac{\xi}{x}\right) \Gamma\left(0, \frac{\xi}{x}\right)\right)}{\log x}\right]}_{\stackrel{(b)}{=} 0} \\
& =K
\end{aligned}
$$

where $x=\mathrm{SNR}, \xi=\frac{K}{g \Omega_{g}}$ is a positive constant, and $(b)$ follows from the Laguerre polynomial series representation of incomplete gamma function [12] together with l'Hôspital rule. This completes the proof.

\section{Numerical Results AND Discussion}

In this section, we validate our analysis by comparing with Monte-Carlo simulation. In the following numerical examples, we consider the non-regenerative relay protocol employing $\mathcal{G}_{2}$ and $\mathcal{G}_{4}$. We assume collinear geometry for locations of three communicating terminals. The path-loss of each link follows an exponential-decay model: if the distance between the source and destination is equal to $d$, then the channel mean power of source-destination link $\Omega_{0} \propto d^{-\alpha}$ where the exponent $\alpha=4$ corresponding to a typical non line-of-sight propagation. Then, $\Omega_{f}=\epsilon^{-\alpha} \Omega_{0}$ and $\Omega_{g}=(1-\epsilon)^{-\alpha} \Omega_{0}$, where $\epsilon \in[0,1]$ is the ratio of the distance of source-relay link and that of sourcedestination link [8].

Fig. 1 shows the SEP of QPSK versus SNR when the relay approaches the the source with $\epsilon=0.3$ when the number of relays is 2,3 , and 4 . We use the one rate OSTBC $\mathcal{G}_{2}$ (Alamouti code) for the case of two relays and a simple 3/4-rate OSTBC $\mathcal{G}_{4}$ for the case of three and four relays. As seen from the figure, analytical and simulated PEP curves match exactly. Observe that the SEP slops, i.e., diversity gain, increases with the number of relays, as speculated in Theorem 1.

\section{CONCLUSION}

In this paper, we have analyzed the SEP of cooperative system, in which the relays generate orthogonal space-time block code using amicable orthogonal designs in a distributed fashion to exploit the benefit of MIMO system in relay fading channels. Specifically, the final expressions can be described in exact closed-form and easily evaluated using common mathematical software packages. We have quantified the effect of SEP in the high SNR regime and shown that the full diversity order can be achieved.

\section{REFERENCES}

[1] J. N. Laneman, D. N. C. Tse, and G. W. Wornell, "Cooperative diversity in wireless networks: Efficient protocols and outage behavior," IEEE Trans. Inf. Theory, vol. 50, no. 12, pp. 3062-3080, Dec. 2004.

[2] J. N. Laneman and G. W. Wornell, "Distributed space-time-coded protocols for exploiting cooperative diversity in wireless networks," IEEE Trans. Inf. Theory, vol. 49, no. 10, pp. 2415-2425, Oct. 2003.

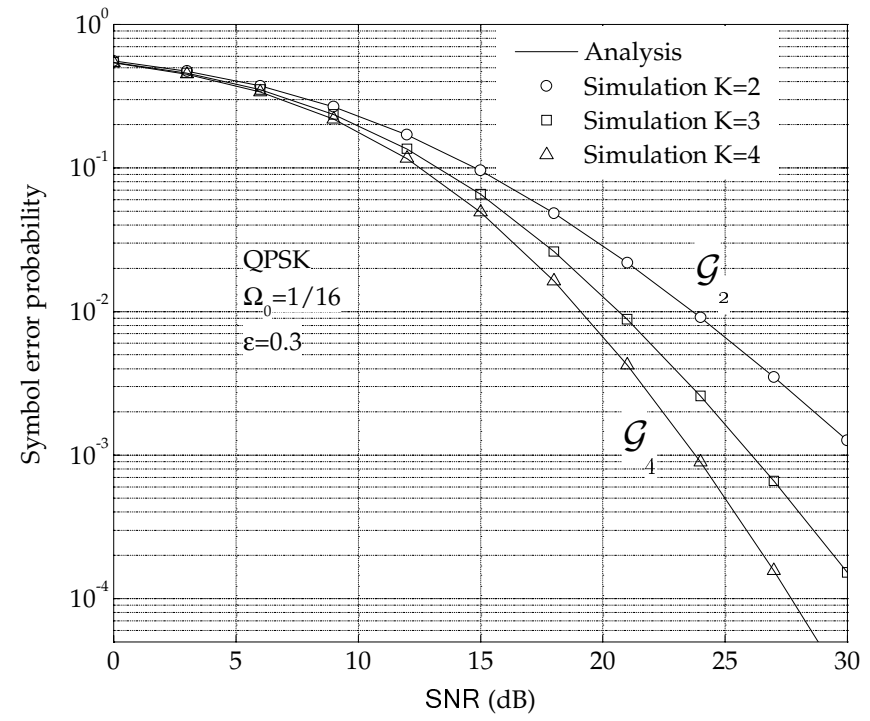

Fig. 1. Symbol error probability of QPSK versus SNR in non-regenerative relay channels employing amicable orthogonal designs when the number of relays is 2,3 , and $4 . \Omega_{0}=\frac{1}{16}$.

[3] Y. Jing and B. Hassibi, "Distributed space-time coding in wireless relay networks," IEEE Trans. Wireless Commun., vol. 5, no. 12, pp. 35243536, Dec. 2006.

[4] Z. Yi and I.-M. Kim, "High data-rate single-symbol ML decodable distributed STBCs for cooperative networks," IEEE Transactions on Information Theory, accepted for publication.

[5] Y. Jing and H. Jafarkhani, "Using orthogonal and quasi-orthogonal designs in wireless relay networks," in Proc. IEEE Global Commununications Conf., San Francisco, CA, Nov-Dec 2006.

[6] V. Tarokh, H. Jafarkhani, and A. R. Calderbank, "Space-time block codes from orthogonal designs," IEEE Trans. Inf. Theory, vol. 45, no. 5, pp. 1456-1467, Jul. 1999.

[7] G. Ganesan and P. Stoica, "Space-time block codes: A maximum SNR approach," IEEE Trans. Inf. Theory, vol. 47, no. 4, pp. 1650-1656, May 2001.

[8] T. Q. Duong, H. Shin, and E.-K. Hong, "Effect of line-of-sight on dualhop nonregenerative relay wireless communications," in Proc. IEEE Veh. Technol. Conf., Batimore, Maryland, Sep. 2007, to be published.

[9] V. Tarokh, H. Jafarkhani, and A. R. Calderbank, "Space-time block coding for wireless communications: Performance results," IEEE J. Sel. Areas Commun., vol. 17, no. 3, pp. 451-460, Mar. 1999.

[10] X. Li, T. Luo, G. Yue, and C. Yin, "A squaring method to simplify the decoding of orthogonal space-time block codes," IEEE Trans. Commun., vol. 49, no. 10, pp. 1700-1703, Oct. 2001.

[11] T. Q. Duong, E.-K. Hong, and H. Shin, "Symbol error probability of distributed-Alamouti scheme with non-regenerative relays," 2007, submitted for publication.

[12] I. S. Gradshteyn and I. M. Ryzhik, Table of Integrals, Series, and Products, 6th ed. San Diego, CA: Academic, 2000.

[13] M. K. Simon and M.-S. Alouini, "A unified approach to the performance analysis of digital communication over generalized fading channels," Proc. IEEE, vol. 86, no. 9, pp. 1860-1877, Sep. 1998.

[14] H. Shin, T. Q. Duong, and E. K. Hong, "MIMO cooperative diversity with amplify-and-forward relaying," IEEE Trans. Commun., 2007, submitted for publication. 